\title{
Highly accelerated Point-Spread Function mapping based on Finite Rate of Innovation for EPI distortion correction
}

\author{
Rita G Nunes ${ }^{1,2^{*}}$, Joseph V Hajnal ${ }^{2,3}$ \\ From PSMR14: 3rd Conference in PET/MR and SPECT/MR \\ Kos Island, Greece. 19-21 May 2014
}

${ }^{1}$ Institute of Biophysics and Biomedical Engineering, Faculty of Sciences, University of Lisbon, Lisbon, Portugal
Hybrid MR-PET scans enable acquisition of both types of images within a session. Despite consistent subject positioning, image alignment can still be challenging. Functional MR images rely on echo-planar imaging (EPI) and present geometric distortions due to static B0 field inhomogeneities. Direct B0 [1] and Point Spread Function (PSF) mapping [2] (Figure 1) have been proposed for distortion correction. The PSF method is more robust [3], but acquisition times are long even with previous acceleration approaches [4-7].

We used the Finite Rate of Innovation (FRI) framework [8,9] to detect the PSF peak position to sub-pixel precision using as few $\mathrm{k}_{\mathrm{s}}$ samples as possible.

Images were acquired on a 3.0T Philips Achieva: $2.5 \times 2.5 \times 4.0 \mathrm{~mm}^{3}$, matrix $96 \times 95,95$ $\mathrm{k}_{\mathrm{s}}$ steps with under-sampling retrospectively performed. PSF peak position was estimated using both the fully-sampled (zero-filled by a factor of 1000) and highly-undersampled data.

To determine the PSF peak location, pattern matching was performed. The signal measured at each spatial location was compared to a predicted signal pattern accounting for the $k_{\mathrm{s}}$ sampling scheme and the search progressively refined up to the intended precision. EPI images were undistorted as in [10].

Figure 2 shows example PSF peak shift maps (relative to expected undistorted positions). Figure 3A shows the original EPI slice matching Figure 2, and Figures 3B-E show the corrected images using each displacement map. Comparison with the GE image (Figure 3F) confirms accurate geometrical corrections.

Using the proposed approach the position of the PSF peak can be estimated from a very small number of samples. In the future distortion map estimation could easily be incorporated into standard preparation phases. Making distortion correction of EPI images more practical would facilitate combining functional PET and MR information as well as structural connectivity information from diffusion-weighted MR images.

\section{SpringerOpen ${ }^{\circ}$}

(C) 2014 Nunes and Hajnal; licensee Springer This is an Open Access article distributed under the terms of the Creative Commons Attribution License (http://creativecommons.org/licenses/by/4.0), which permits unrestricted use, distribution, and reproduction in any medium, provided the original work is properly cited. 


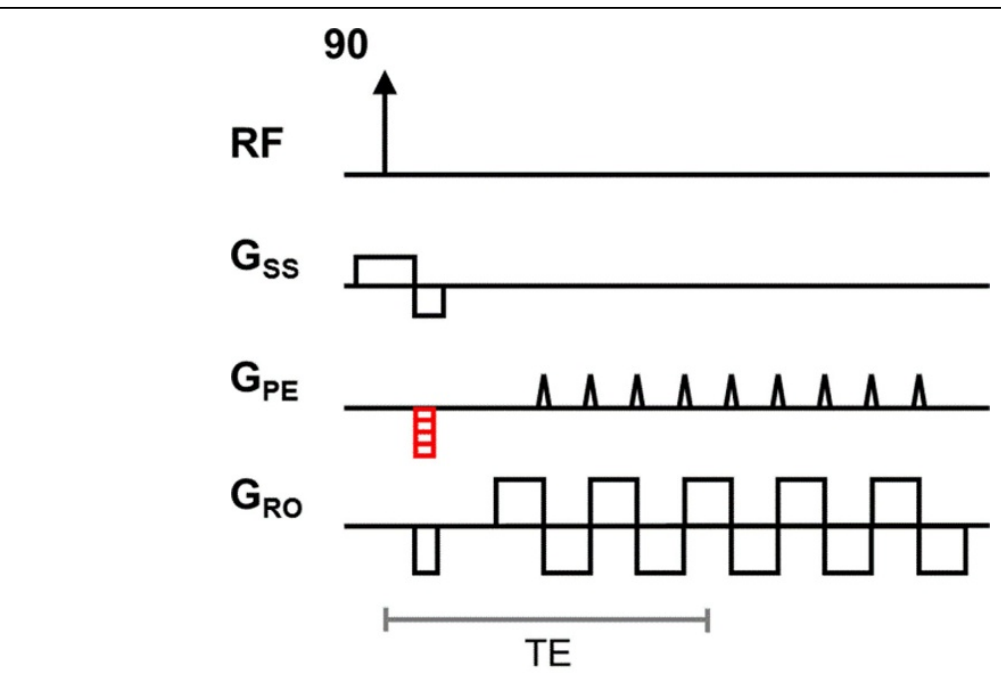

Figure 1 The PSF mapping sequence is repeated while varying the amplitude of the prewinder phase encode gradient (in red).

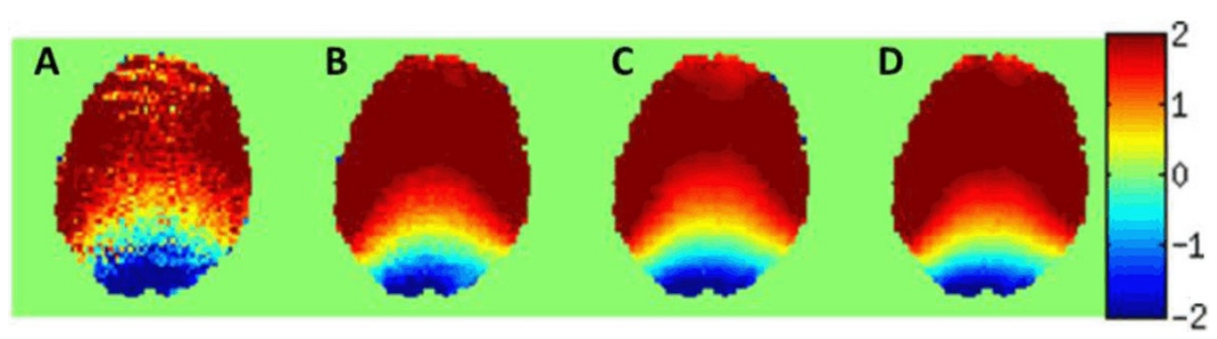

Figure 2 Maps of the PSF shifts (in pixels) estimated with: A0 2; B) 3; C) 4; D) all $95 k_{s}$

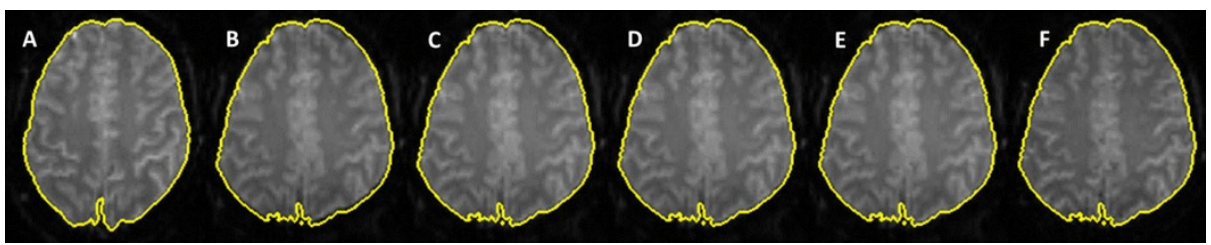

Figure $3 \mathrm{EPI}$ image (with corresponding outer contour in yellow); Undistorted EPI images estimating the displacement field from: B) 2; C) 3; D) 4 and E) all $95 \mathrm{k}_{\mathrm{s}}$ samples and F) GE image (corresponding outer contour in yellow propagated to all undistorted EPI images)

\section{Authors' details}

${ }^{1}$ Institute of Biophysics and Biomedical Engineering, Faculty of Sciences, University of Lisbon, Lisbon, Portugal. ${ }^{2}$ Division of Imaging Sciences and Biomedical Engineering, King's College London, London, UK. ${ }^{3}$ Centre for the Developing Brain, King's College London, London, UK.

Published: 29 July 2014

\section{References}

1. Jezzard P, et al: Correction for geometric distortion in echo planar images from B0 field variations. MRM 1995, 34:65-73.

2. Robson MD, et al: Measurement of the point spread function in MRI using constant time imaging. MRM 1997, $38: 733$.

3. Zeng $\mathrm{H}$, et al: Image distortion correction in EPI: Comparison of field mapping with point spread function mapping. MRM 2002, 48:137-146. 
4. Zaitsev $M$, et al: Point spread function mapping with parallel imaging techniques and high acceleration factors: Fast, robust, and flexible method for echo-planar imaging distortion correction. MRM 2004, 52:1156-66.

5. In $\mathrm{M}$, et al: Highly accelerated PSF-mapping for EPI distortion correction with improved fidelity. MagnResonMaterPhy 2012, 25:183-192

6. Dragonu I, et al: Accelerated point spread function mapping using signal modeling for accurate echo-planar imaging geometric distortion correction. MRM 2013, 69:1650-56.

7. Nunes RG, et al: ESMRMB 2013, 30:145.

8. Vetterli M, et al: Sampling signals with finite rate of innovation. IEEE Trans Sig Process 2002, 50:1417-28.

9. Maravic I, et al: IEEE Trans Sig Process 2005, 53:2788-2805.

10. Oh SH, et al: Distortion correction in EPI at ultra-high-field MRI using PSF mapping with optimal combination of shift detection dimension. MRM 2012, 68:1239-46.

doi:10.1186/2197-7364-1-S1-A45

Cite this article as: Nunes and Hajnal: Highly accelerated Point-Spread Function mapping based on Finite Rate of Innovation for EPI distortion correction. EJNMMI Physics 2014 1(Suppl 1):A45.

\section{Submit your manuscript to a SpringerOpen ${ }^{\circ}$} journal and benefit from:

Convenient online submission

- Rigorous peer review

- Immediate publication on acceptance

- Open access: articles freely available online

- High visibility within the field

- Retaining the copyright to your article

Submit your next manuscript at $>$ springeropen.com 